\title{
IncRNA $H 19$ acts as a ceRNA to regulate the expression of CTGF by targeting miR-19b in polycystic ovary syndrome
}

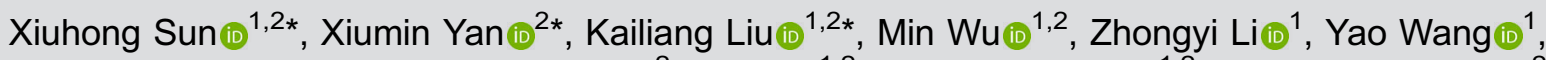

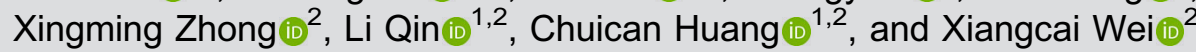 \\ ${ }^{1}$ School of Medicine, Jinan University, Guangzhou, Guangdong Province, China \\ ${ }^{2}$ Guangdong Women and Children Hospital, Guangzhou, Guangdong Province, China
}

\begin{abstract}
The etiology of polycystic ovary syndrome (PCOS) is complex and the pathogenesis is not fully understood. Some studies have shown that dysregulation of ovarian granulosa cells may be related to abnormal follicles and excessive androgen in women with PCOS. Our team has also confirmed the high expression status of $H 19$ in PCOS patients in the early stage. However, the relationship between $\mathrm{H} 19$ and miR-19b in the development of PCOS is still unknown. Therefore, we used bioinformatics to predict the binding sites of human $\mathrm{H} 19$ and miR-19b, and of miR-19b and CTGF genes. After the silencing and overexpression of $H 19$, real-time polymerase chain reaction (PCR) was used to detect the expressions of H19, miR-19b, and CTGF. Western blotting was used to detect CTGF protein. Proliferation of KGN cells after $H 19$ silencing was detected by CCK8. Flow cytometry was used to detect the apoptosis of KGN cells after $H 19$ silencing. After the overexpression of $H 19$, it was found that the expression of miR-19b gene decreased and the expression of CTGF increased, whereas silencing of H19 did the opposite. In addition, $\mathrm{H} 19$ could promote cell proliferation and decrease cell apoptosis. Finally, luciferase reporter assays showed that the $3^{\prime}$-end sequences of IncRNA H19 and CTGF contained the binding site of miR-19b. In conclusion, our study indicated that IncRNA H19 acted as a ceRNA to bind to miR-19b via a "sponge" to regulate the effect of CTGF on KGN cells, which may play a vital role in PCOS.
\end{abstract}

Key words: Polycystic ovary syndrome; Long non-coding RNA H19; microRNA-19b; CTGF

\section{Introduction}

Polycystic ovary syndrome (PCOS) is a highly heterogeneous and complex disease that is one of the most common reproductive endocrine diseases in gynecology (1). It affects both birth and metabolism. Its incidence is rising, and the number of patients is large, reaching $5-10 \%$ of women of childbearing age (2). The etiology and pathogenesis of PCOS have become hotspots in many disciplines such as obstetrics and gynecology, endocrinology, and reproductive science. Although the exact cause of PCOS is still not fully understood, the survival and proliferation of granulosa cells may be the cause of PCOS (3). There is evidence that dysfunction of granulosa cells may lead to the formation of abnormal follicles in PCOS patients, but the specific mechanism remains to be determined. For PCOS, a large number of studies have confirmed that CTGF is highly expressed in the ovaries of PCOS patients and PCOS animal models (4).
Experiments have confirmed that CTGF is involved in PCOS granulosa cell proliferation, and high expression of CTGF promotes granulosa cell proliferation and inhibits apoptosis (5).

Noncoding RNA (ncRNA) is an RNA that does not encode a protein in the body. In the last decade, with the development of sequencing technology, most RNA transcripts do not encode proteins. Depending on the number of RNAs, it can be divided into short non-coding RNAs (such as miRNAs) and long non-coding RNAs (such as IncRNA, circRNA, etc.) (6). MicroRNAs (miRNAs) are a class of highly conserved, non-coding, short-stranded RNAs that have approximately 20-23 nucleotides in length. miRNAs regulate post-transcriptionality by base pairing with the $3^{\prime}$ untranslated region (UTR) of the target mRNA (7). Horizontal mRNA transcription and translation affect biological function. IncRNAs are a class of RNAs

Correspondence: Xiangcai Wei: <Dxcwei@163.com>

${ }^{*}$ These authors contributed equally to this work. 
that are more than 200 nucleotides in length and have limited protein-coding potential (8). Studies have shown that IncRNAs have many functions in various pathophysiological processes. The dysfunction of IncRNAs plays a key role in many diseases. The function of IncRNA is complex. In 2011, Karreth et al. (9) proposed a theory called competitive endogenous RNA (ceRNA) hypothesis that IncRNAs, mRNAs, and pseudogenes can competitively bind to microRNA response elements (MRE) and lead to mutual regulation of expression, which provides a new gene regulation mechanism.

In recent years, in order to more deeply explore the etiology of PCOS from the epigenetics, researchers are beginning to pay attention to the relationship between non-coding RNAs and PCOS. Huang et al. (10) reported that IncRNA H19 competes with STAT3 for binding to miR19, which in turn affects STAT3 expression. Zhong et al. (11) found that in PCOS, miR-19b is lowly expressed in ovarian tissue and granulosa cells, and miR-19b can regulate the proliferation of granulosa cells. However, there are no reports on the interaction between H19 and miR-19b in PCOS. Therefore, this study focused on the relationship between $\mathrm{H} 19$ and miR-19b in KGN cells, its effect on gene CTGF, and explored the role of $H 19$ in the development of PCOS.

\section{Material and Methods}

\section{Cell recovery and culture}

A thermostatic water bath was preheated to $37^{\circ} \mathrm{C}$, and the table top was wiped and ultra-cleaned with $75 \%$ ethanol beforehand. Then, an ultraviolet light was turned on to illuminate the table for $30 \mathrm{~min}$. The cryotube was quickly placed into a $37^{\circ} \mathrm{C}$ constant temperature water bath, and shaken continuously, so that the cell cryopreservation medium in the cryotube was melted rapidly and taken out. The tube was centrifuged at $800 \mathrm{~g}$ for $5 \mathrm{~min}$ at $4^{\circ} \mathrm{C}$; the supernatant was discarded, $1-\mathrm{mL}$ of medium was added, and the cells were shaken as appropriate to resuspend the cells. The cells were then placed in culture dishes, which were placed in a carbon dioxide incubator at $37^{\circ} \mathrm{C}$ for constant temperature culture for $48 \mathrm{~h}$, and passed 2-3 generations to prove that the cells were viable. The culture solution was continuously changed according to the growth of the cells. The cells were rinsed in the cell culture flask 1-2 times with PBS water. A 1.5-mL trypsin-EDTA solution was added to the flask to lightly wash the cell culture dish. The trypsinEDTA solution was discarded and the cells digested in a $37^{\circ} \mathrm{C}$ carbon dioxide incubator for $3 \mathrm{~min}$. If cells were found to be in a circular state, $1-\mathrm{mL}$ of medium was added for further digestion. The cell solution was repeatedly pipetted with a 1-mL pipette to mix the cells, and the culture solution of each flask was replenished, transferred to a new flask, and the culture solution was added in a diluted ratio.

\section{Real-time PCR}

One millileter of Trizol was added to the cells, and the tube was shaken and mixed at $4^{\circ} \mathrm{C}$ for $5 \mathrm{~min}$. Chloroform $(0.2 \mathrm{~mL})$ was added, the tube was vigorously shaken for $15 \mathrm{~s}$, and then allowed to stand at $4^{\circ} \mathrm{C}$ for $3 \mathrm{~min}$. An equal volume of isopropanol was added, mixed, and left to stand at $-20^{\circ} \mathrm{C}$ for $20 \mathrm{~min}$; the tube was then centrifuged at $12,000 \mathrm{~g}$ for $15 \mathrm{~min}$ at $4^{\circ} \mathrm{C}$, and the supernatant removed. Centrifugation was repeated at $8000 \mathrm{~g}$ for $5 \mathrm{~min}$ at $4^{\circ} \mathrm{C}$, and $1.5 \mu \mathrm{L}$ of the sample solution was measured for concentration in an ultra-micro UV analyzer. Reverse transcription was performed as described in the Bestar ${ }^{\mathrm{TM}}$ qPCR RT Kit (DBI, Germany).

\section{Cell transfection}

KGN cells $(80 \%)$ were removed and the old medium was aspirated. The cells were washed one or two times with PBS, trypsin solution was added, and then aspirated when the cells were separated and round pellets appeared. Fresh complete medium was added, and the cells were pipetted well to prepare a single-cell suspension. The cell density was adjusted to $8 \times 10^{4} / \mathrm{mL}$ and $0.25 \mu \mathrm{L}$ of siRNA or $0.2 \mu \mathrm{g}$ of plasmid was added to $50 \mu \mathrm{L}$ of serumfree medium, gently mixed, and allowed to stand at room temperature for $5 \mathrm{~min}$. Lipofectamine ${ }^{\mathrm{TM}} 2000(0.25 \mu \mathrm{L}$; Thermo Fisher Scientific, USA) was added to $50 \mu \mathrm{L}$ of serum-free medium, gently mixed, and allowed to stand at room temperature for $5 \mathrm{~min}$. The diluted siRNA or plasmid was mixed with Lipofectamine ${ }^{T M} 2000$, gently mixed, and allowed to stand at room temperature for $20 \mathrm{~min}$ to form a plasmid/Lipofectamine ${ }^{T M} 2000$ complex. siRNA $(100 \mu \mathrm{L})$ or plasmid/Lipofectamine ${ }^{\mathrm{TM}} 2000$ complex was added to the wells of the corresponding group of culture plates at a final concentration of $50 \mathrm{nM}$; after $6 \mathrm{~h}$, the cells were replaced with complete medium for $48 \mathrm{~h}$. The samples were subjected to qPCR and western blot detection to screen for optimal sequences.

\section{Cell proliferation}

Cell viability was determined using Cell Counting Kit-8 (CCK8) (Dojindo, Japan) according to the instructions of the manufacturer. In brief, KGN cells during logarithmic growth period were seeded into 96 -well microtiter plates at a density of $5 \times 10^{3}$ cells/well. Then, cells were transfected with indicated plasmids or oligonucleotides. After transfection for $48 \mathrm{~h}, 10 \mu \mathrm{L}$ of CCK-8 reagent was added to each well and incubated for another $4 \mathrm{~h}$ at $37^{\circ} \mathrm{C}$ and $5 \% \mathrm{CO}_{2}$. Absorbance at $450 \mathrm{~nm}$ was measured to assess relative cell viability using a microplate reader (Bio-Rad, USA).

\section{Apoptosis}

Cell apoptosis was examined using an Annexin V-FITC apoptosis detection kit (Abcam, UK) following the manufacturer's instructions. KGN cells at a density of $1 \times 10^{5}$ cells/well were rinsed twice with cold PBS solution and 
resuspended in $1 \times$ binding buffer. Afterwards, $5 \mu \mathrm{L}$ of Annexin V-FITC and $10 \mu \mathrm{L}$ of propidium iodide (PI) were introduced and incubated for $10 \mathrm{~min}$ in the dark. Finally, apoptotic cells were determined using a flow cytometer (BD Biosciences, USA)

\section{Luciferase reporter assay}

Bioinformatics analysis using Starbase online software (Sun Yat-sen University, China) first proved the existence of putative binding sites between $\mathrm{H} 19$ and miR-19b. For luciferase assay, partial fragments of $H 19$ containing the assumed miR-19b binding sites were amplified and sub-cloned into psiCHECK-2 vector (Promega, USA) to generate wild-type $\mathrm{H} 19$ plasmid (H19-WT). Then, a SiteDirected Mutagenesis Kit (Thermo Fisher Scientific) was used to generate mutated $\mathrm{H} 19$ plasmid (H19-MUT). H19WT and H19-MUT constructs were co-transfected into KGN cells along with miR-19b mimics or miR-NC (negative control). About 48-h post-transfection, relative luciferase activity was determined using the dual-luciferase reporter assay system (Promega).

\section{Statistical analysis}

All experiments were performed three times. Statistical analysis was conducted using SPSS 20.0 (IBM Corp., USA), and results are reported as means \pm SD. Student's $t$-test or one-way analysis of variance was carried out to estimate significant group differences. $\mathrm{P}<0.05$ represented a statistically significant result.

\section{Results}

\section{H19 overexpression recombinant plasmid and H19 silencing site}

Total RNA was extracted from KGN cells, and then reversed transcribed into cDNA. PCR amplified $\mathrm{H} 19$ by designed primers, and the product was electrophoresed, and the amplified product was 2362 bp (Figure 1A). H19 was connected to pcDNA3.0 + vector to construct H19pcDNA $3.0+$ recombinant vector and transfer it to $\mathrm{DH} 5 \alpha$ competent cells. Monoclonal colonies were selected and restriction enzymes were used to digest the recombinant vector for PCR identification (Figure 1B).

Three silent sites, 1552, 1617, and 1712, were selected to silence $\mathrm{H} 19$, and the corresponding siRNA was designed and transfected into KGN cells. Compared with the control group, the expression of $\mathrm{H} 19$ was significantly decreased after silencing of $\mathrm{H} 19$ by siH19-1552, siH19-1617, and siH19-1712 $(\mathrm{P}<0.05)$. Among the three silent sites, the expression of siH19-1617 was significantly more pronounced after silencing $\mathrm{H} 19$, and the silencing effect was the best of the three (Figure 1C).

\section{Overexpression of IncRNA H19 in KGN cells promoted the expression of CTGF}

After transfecting the constructed $\mathrm{H} 19$ overexpression plasmid into KGN cells, H19, miR-19b, and CTGF were detected at the RNA level and protein expression by qRT-PCR and Western blot in the presence of H19 overexpression.

Compared with the pcDNA3.0 + NC group, the expression of $\mathrm{H} 19$ transfected with the $\mathrm{H} 19$ overexpression plasmid group was significantly increased $(P<0.05)$ (Figure 2A). Compared with the pcDNA3.0 + NC group, miR-19b transfected with the $\mathrm{H} 19$ overexpression plasmid group was significantly decreased $(P<0.05)$ (Figure $2 B$ ). Compared with the pcDNA3.0 + NC group, CTGF transfected with the H19 overexpression plasmid group was significantly increased $(\mathrm{P}<0.05)$ (Figure $2 \mathrm{C}$ ). The expression of CTGF protein was consistent with the expression of CTGF at the RNA level. After H19 overexpression, the expression of CTGF protein was also significantly increased (Figure 2D).

\section{Overexpression of $\boldsymbol{H} 19$ promoted KGN cell proliferation and inhibited KGN cell apoptosis}

Compared with the control group, KGN cells in the overexpressed group had enhanced proliferative capacity at 48 and $72 \mathrm{~h}$, and the apoptotic ability was weakened (Figure 3, Supplementary Figure S1). The results indicated
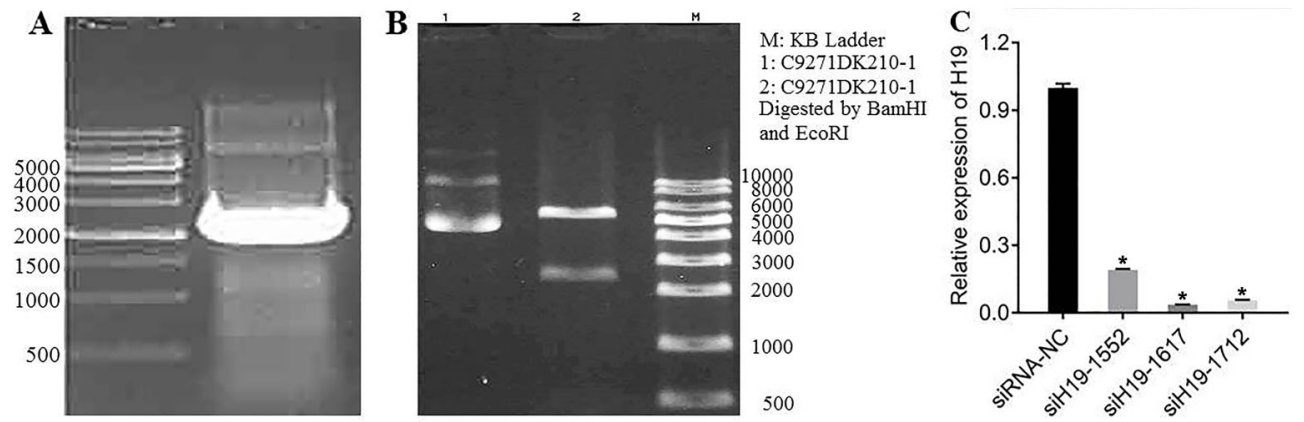

Figure 1. A, H19 PCR amplification electropherogram. B, Restriction endonuclease digestion of recombinant vector. C, Changes in $\mathrm{H} 19$ expression after silencing $\mathrm{H} 19$. Data are reported as means $\pm \mathrm{SD}$. ${ }^{*} \mathrm{P}<0.05$ compared to negative control (NC) (one-way analysis of variance). 


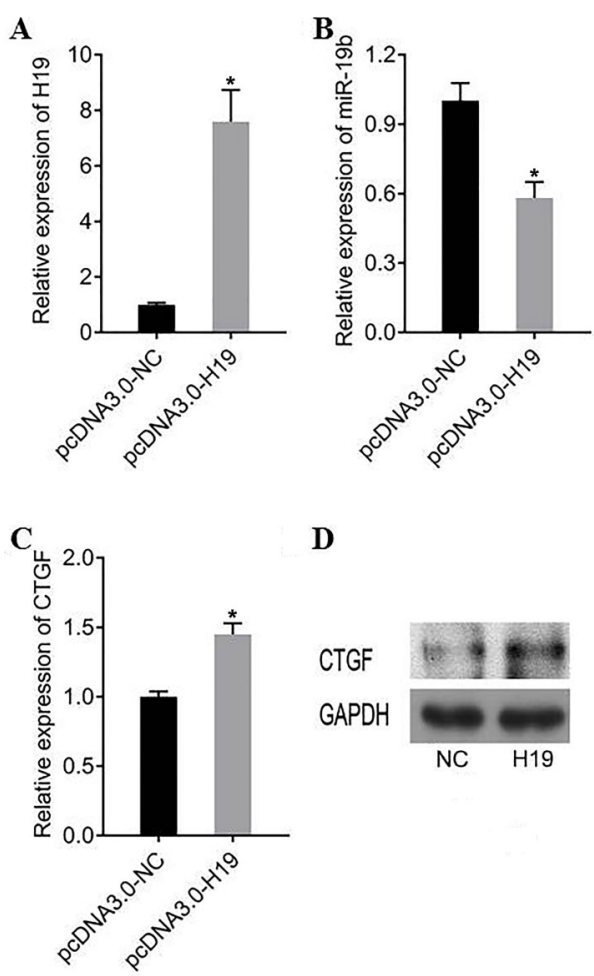

Figure 2. A, H19 expression after overexpression of $H 19$. B, Expression of miRN-19b after overexpression of H19. C and D, CTGF expression after overexpression of H19. Data are reported as means $\pm S D$. ${ }^{*} \mathrm{P}<0.05$ compared to negative control (NC) $(t$-test).

that high expression of $\mathrm{H} 19$ had an effect on promoting proliferation and inhibiting apoptosis in KGN cells.

\section{Silencing IncRNA H19 in KGN cells inhibited the expression of CTGF}

Compared with the corresponding siRNA control group, the expression of miR-19b in KGN cells transfected with siH19-1617 was significantly increased $(P<0.05)$. The CTGF reduction of KGN cells transfected with siH191617 was significant $(P<0.05)$ compared to the corresponding siRNA control group. The CTGF protein results were consistent with the results of qRT-PCR. After silencing $H 19$, the expression of CTGF protein was decreased (Figure 4A-C).

\section{Low expression of $\mathrm{H} 19$ inhibited KGN cell proliferation} and promoted KGN cell apoptosis

Compared with the siRNA control group, after transfection of siH19-1617 to KGN cells, the proliferation of KGN cells was weakened at 48 and $72 \mathrm{~h}$, and apoptosis was enhanced (Figure 4D-E, Supplementary Figure S2). The experimental results showed that the low expression of $\mathrm{H} 19$ inhibited the proliferation of KGN cells and promoted the apoptosis of KGN cells.
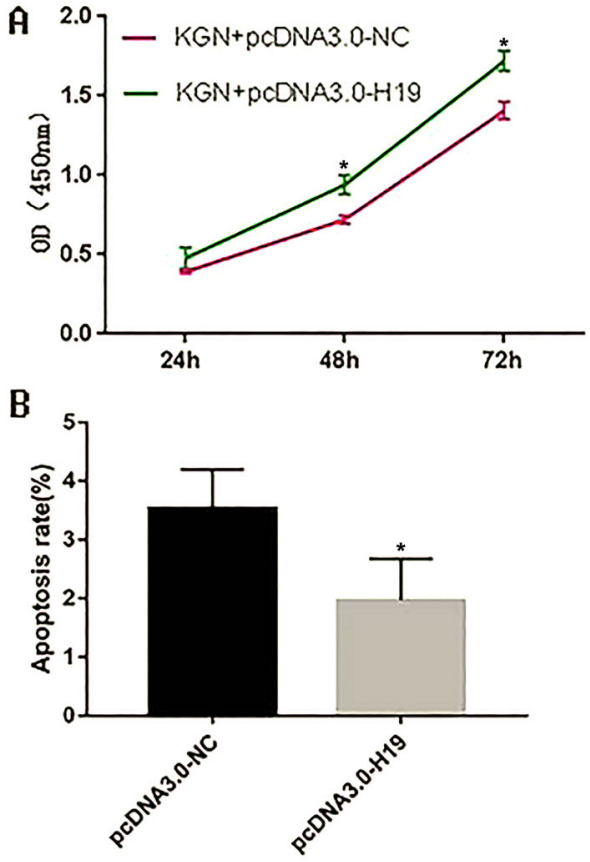

Figure 3. A, Cell proliferation was detected by CCK8 at 24, 48, and $72 \mathrm{~h}$ in KGN cells overexpressing H19. B, Detection of KGN cell apoptosis by flow cytometry after overexpressing H19. Data are reported as means $\pm S D$. ${ }^{*} \mathrm{P}<0.05$ compared to negative control (NC) ( $t$-test).

\section{Dual luciferase reporter gene}

Compared with the control group, the miR-19b mimics co-transfected with wild-type $\mathrm{H} 19$ or $3^{\prime}$ UTR CTGF inhibited the fluorescence activity of $\mathrm{H} 19$ and CTGF $(P<0.05)$. After mutation of the binding site sequence, miR-19b mimics had no effect on the fluorescence activity of the mutant $H 19$ and CTGF $(\mathrm{P}>0.05)$. The results of the dual luciferase reporter gene showed that the binding mode between IncRNA H19 and miR-19b and between miR-19b and CTGF were both directly combined targeted adsorption (Figure 5).

\section{Discussion}

PCOS is a common reproductive endocrine disease in gynecology. Anovulatory infertility caused by PCOS is a serious problem for women of childbearing age (12). PCOS not only affects female fertility but also affects multiple organs in the body $(13,14)$. Currently, there is no effective drug to cure PCOS completely, and many patients suffer from PCOS for life. The pathogenesis of polycystic ovary syndrome is complex, and in-depth study of its specific molecular regulation mechanism can provide a useful target for the diagnosis and treatment of PCOS.

In our previous study, we found that IncRNA H19 was highly expressed in peripheral blood of patients with PCOS (15). There are many studies on CTGF in PCOS 
A
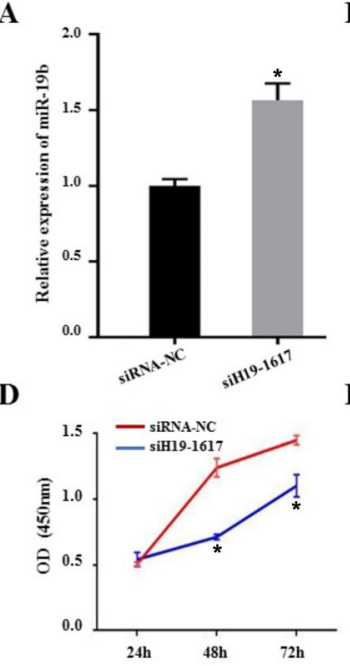

B
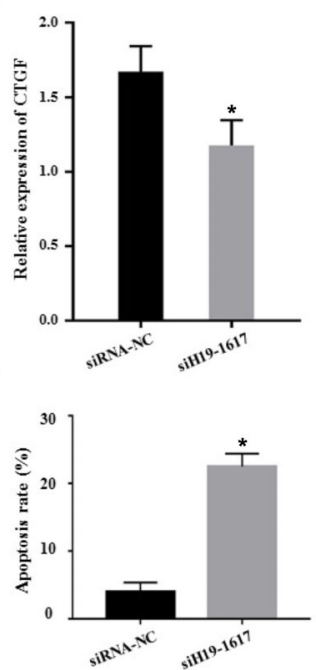

C

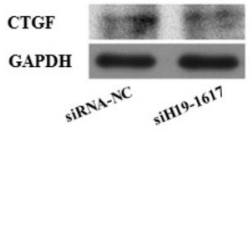

Figure 4. A, Expression of miR-19b after silencing H19. B, CTGF expression after silencing H19. C, Expression of CTGF protein after silencing H19. D, The proliferative ability of KGN cells after silencing $H 19$ was detected by CCK8 at 24,48 , and $72 \mathrm{~h}$. E. KGN cells apoptosis after silencing $\mathrm{H} 19$ was detected by flow cytometry. ${ }^{*} \mathrm{P}<0.05$ compared to negative control (NC). Data are reported as means $\pm \mathrm{SD}$ ( $t$-test).
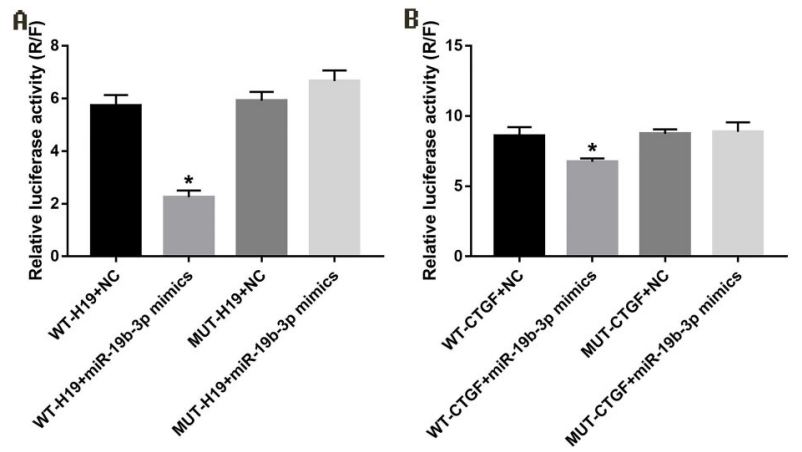

Figure 5. A, IncRNA $H 19$ and miR-19b dual luciferase reporter gene test results $\left({ }^{*} \mathrm{P}<0.05\right.$ vs WT-H19+NC). B, CTGF and miR-19b dual luciferase reporter gene test results $\left({ }^{*} \mathrm{P}<0.05\right.$ vs WT-CTGF $+N C)$. Data are reported as means $\pm S D$ (t-test). NC: negative control; WT: wild type; MUT: mutant type.

that confirm that CTGF is highly expressed in PCOS (16), but the reason why is unknown. Is there an upstream regulatory factor of the highly expressed CTGF? Is noncoding RNA involved in the regulation of CTGF expression? What role does IncRNA H19 play in the regulation of CTGF expression? These issues require in-depth study.

Follicular dysplasia can be observed in patients with PCOS, and granulosa cells around the follicle play a key role in follicular development (17). An important clinical feature of PCOS is infertility caused by abnormal follicular development in women of childbearing age, which is also the main cause of PCOS in patients (17). During growth and development of the oocyte, the oocyte is closely related to the surrounding granulosa cells (18). The nutrients during the growth and development of the oocyte are provided by the surrounding granulosa cells, and the granulosa cells provide growth regulators to promote oocyte maturation (19). The ovaries of PCOS patients contain twice as many follicles at various stages of development. The number of granulosa cells in the ovary of anovulatory PCOS patients is increased compared to the number of granulosa cells in normal ovaries. These conclusions are also confirmed in the PCOS animal model. The relationship between CTGF and granulosa cell proliferation phenotype involved in this experiment has been clarified. High expression of CTGF can promote granulosa cell proliferation and inhibit granulosa cell apoptosis (20).

The experimental results of our KGN cell study showed that overexpression of $H 19$ promoted the expression of CTGF and inhibited the expression of miR-19b. By inhibiting the expression of $H 19$ by siRNA, the expression of CTGF can be inhibited and the expression of miR-19b can be promoted. CTGF protein level also confirmed the results of changes in CTGF at the RNA level. We studied the effects of IncRNA H19 on proliferation and apoptosis of KGN cells by CCK8 and flow cytometry. With overexpression of $H 19, \mathrm{KGN}$ cells increased proliferation and decreased apoptosis; with silencing of $H 19, \mathrm{KGN}$ cells decreased proliferation and increased apoptosis. The results showed that IncRNA H19 can affect the downstream molecular CTGF in KGN cells, and abnormally expressed CTGF can participate in the pathogenesis of PCOS granulosa cell proliferation and ovarian fibrosis. IncRNA H19 ultimately affected the pathogenesis of 
PCOS by regulating the expression of CTGF. We found that the direction of regulation of IncRNA H19 was consistent with that of CTGF. With overexpression of IncRNA, H19 and CTGF were also highly expressed. The regulation of IncRNA H19 was opposite to that of miR-19b. After overexpression of IncRNA H19, miR-19b expression was decreased. After silencing IncRNA H19, the situation also satisfied the above. This regulatory trend was consistent with ceRNA. In ceRNA, the regulation trend of IncRNA and mRNA was the same, and the regulation trend of IncRNA and miRNA was the opposite. This provides a basis for our subsequent research on ceRNA mechanisms.

In order to solve the hypothesis that IncRNA H19 directly binds to $m i R-19 b$ via ceRNA mechanism to regulate CTGF, we verified by dual luciferase assay that miR-19b directly targeted IncRNA H19 and CTGF. Our study showed that IncRNA $H 19$ can bind to miR-19b, and the binding ability of $m i R-19 b$ and CTGF was reduced, which reduced the inhibition of CTGF gene expression by $m i R-19 b$, thereby promoting the expression of CTGF. In conclusion, IncRNA H19 affected the expression of CTGF by competitively binding $m i R-19 b$, and this abnormally expressed CTGF was involved in KGN miR-19b cells proliferation.

In this study, we found that was an intermediate molecule in the IncRNA H19/miR-19b/CTGF regulatory axis. IncRNA $H 19$ regulated CTGF expression via the 'intermediate bridge' miR-19b. miR-19b acted as a 'middleman' in this ceRNA regulatory axis.

This is the first time that the proliferation of granulosa cells from the direction of ceRNA regulation mechanisms was explored. In previous studies, the researchers found that miRNAs can regulate the downstream target gene mRNA, which affects granulosa cell proliferation. In this experiment, we further explored the upstream molecules, finding that IncRNA-miRNA-mRNA regulatory axis regulated the proliferation of granulosa cells.

In summary, this experiment confirmed the role of IncRNA $H 19$ as a ceRNA in KGN cells, which regulated

\section{References}

1. Gateva AT, Velikova TV, Kamenov ZA. Peroxiredoxin 4 levels in patients with PCOS and/or obesity. J Gynecol Obstet Hum Reprod 2019; 48: 739-743, doi: 10.1016/ j.jogoh.2019.04.002.

2. He $Y, L u Y$, Zhu Q, Wang $Y$, Lindheim SR, Qi J, et al. Influence of metabolic syndrome on female fertility and in vitro fertilization outcomes in PCOS women. Am J Obstet Gynecol 2019; 221: 138.e1-138.e12, doi: 10.1016/j.ajog. 2019.03.011.

3. Li Y, Xiang Y, Song Y, Wan L, Yu G, Tan L. Dysregulated miR-142, $-33 b$ and -423 in granulosa cells target TGFBR1 and SMAD7: a possible role in polycystic ovary syndrome. $\mathrm{Mol}$ Hum Reprod 2019; 25: 638-646, doi: 10.1093/molehr/gaz014. the expression of CTGF through the IncRNA H19/miR$19 \mathrm{~b} / C T G F$ regulatory axis, and finally played an important role in the pathogenesis of PCOS. However, it should be noted that the ceRNA regulatory network is complex $(21,22)$. Each molecule that can act as a ceRNA (such as IncRNA, circRNA, etc.) contains multiple MREs, which can simultaneously bind to multiple different miRNAs, and the miRNAs ultimately regulate different downstream mRNAs. The regulatory axis has complex biological functions (23). IncRNA H19 is one of the long non-coding RNAs, which was one of the first discovered IncRNAs. Experiments have been carried out to show the new role of IncRNA H19 in ceRNA regulatory networks (24), further confirming the complexity and diversity of ceRNA regulatory networks. Therefore, in PCOS, further exploration of IncRNA H19 and biological phenotypes will help us to understand the pathogenesis of PCOS from a macro perspective.

\section{Supplementary Material}

Click here to view [pdf].

\section{Acknowledgments}

This work was supported in part by China Population Welfare Foundation (grant number: [2011]31), Science and Technology Planning Project of Guangdong Province (grant numbers: 2013B032000001; 2014A020212546), Family Planning Foundation of Guangdong Province (grant number: 2012004), National Natural Science Foundation of China (grant numbers: 30901249; 81101267; 81541070), the Guangdong Natural Science Foundation (grant numbers: 10151063201000036; S2011010002526), Guangdong Science and Technology Plan Project (grant number: 2014A020212229), Guangdong Natural Science Foundation (grant number: 2016A030313760), and Guangzhou Science and Technology Plan Project (grant number: 201804010003).

4. Wang F, Zhang ZF, He YR, Wu HY, Wei SS. Effects of dipeptidyl peptidase-4 inhibitors on transforming growth factor-beta1 signal transduction pathways in the ovarian fibrosis of polycystic ovary syndrome rats. J Obstet Gynaecol Res 2019; 45: 600-608, doi: 10.1111/jog.13847.

5. Gao X, ZY, Li X. Expression of CTGF in polycystic ovary syndrome and its effect on proliferation and apoptosis of ovarian granulosa cells [in Chinese]. J Clin Exp Med 2017; 1686-11689.

6. Liu S, Zhang W, Liu K, Liu Y. LncRNA SNHG16 promotes tumor growth of pancreatic cancer by targeting miR-218-5p. Biomed Pharmacother 2019; 114: 108862, doi: 10.1016/ j.biopha.2019.108862. 
7. Luo W, Yan D, Song Z, Zhu X, Liu X, Li X, et al. miR-126-3p sensitizes glioblastoma cells to temozolomide by inactivating Wnt/beta-catenin signaling via targeting SOX2. Life Sci 2019; 226: 98-106, doi: 10.1016/j.Ifs.2019.04.023.

8. Chu P, Wang Q, Wang Z, Gao C. Long non-coding RNA highly up-regulated in liver cancer protects tumor necrosis factor-alpha-induced inflammatory injury by down-regulation of microRNA-101 in ATDC5 cells. Int Immunopharmacol 2019; 72: 148-158, doi: 10.1016/j.intimp.2019.04.004.

9. Karreth FA, Tay Y, Perna D, Ala U, Tan SM, Rust AG, et al. In vivo identification of tumor- suppressive PTEN ceRNAs in an oncogenic BRAF-induced mouse model of melanoma. Cell 2011; 147: 382-395, doi: 10.1016/j.cell.2011.09.032.

10. Huang Z, Lei W, Hu HB, Zhang H, Zhu Y. H19 promotes non-small-cell lung cancer (NSCLC) development through STAT3 signaling via sponging miR-17. J Cell Physiol 2018; 233: 6768-6776, doi: 10.1002/jcp.26530.

11. Zhong Z, Li F, Li Y, Qin S, Wen C, Fu Y, et al. Inhibition of microRNA-19b promotes ovarian granulosa cell proliferation by targeting IGF-1 in polycystic ovary syndrome. Mol Med Rep 2018; 17: 4889-4898, doi: 10.3892/mmr.2018.8463.

12. Gomez AM, Arteaga S, Ingraham N, Arcara J. Medical conditions, pregnancy perspectives and contraceptive decision-making among young people: an exploratory, qualitative analysis. Contraception 2019; 100: 72-78, doi: 10.1016/ j.contraception.2019.03.046.

13. Hopkins D, Wilson C. Polycystic ovary syndrome in active duty service women: a retrospective analysis. Mil Med 2019; 184: 440-446, doi: 10.1093/milmed/usz023.

14. Schneider D, Gonzalez JR, Yamamoto M, Yang J, Lo JC. The association of polycystic ovary syndrome and gestational hypertensive disorders in a diverse community-based cohort. J Pregnancy 2019; 2019: 9847057, doi: 10.1155/ 2019/9847057.

15. Qin L, Huang CC, Yan XM, Wang Y, Li ZY, Wei XC. Long non-coding RNA H19 is associated with polycystic ovary syndrome in Chinese women: a preliminary study. Endocr $J$ 2019; 66: 587-595, doi: 10.1507/endocri.EJ19-0004.

16. Wang $D$, Wang $W$, Liang $Q$, He X, Xia $Y$, Shen S, et al. DHEA-induced ovarian hyperfibrosis is mediated by TGF-beta signaling pathway. J Ovarian Res 2018; 11: 6, doi: 10.1186/ s13048-017-0375-7.

17. Sagvekar P, Kumar P, Mangoli V, Desai S, Mukherjee S. DNA methylome profiling of granulosa cells reveals altered methylation in genes regulating vital ovarian functions in polycystic ovary syndrome. Clin Epigenetics 2019; 11: 61, doi: 10.1186/s13148-019-0657-6.

18. Shen M, Li T, Zhang G, Wu P, Chen F, Lou Q, et al. Dynamic expression and functional analysis of circRNA in granulosa cells during follicular development in chicken. BMC Genomics 2019; 20: 96, doi: 10.1186/s12864-019-5462-2.

19. Bertoldo MJ, Cheung MY, Sia ZK, Agapiou D, Corley SM, Wilkins MR, et al. Non-canonical cyclic AMP SMAD1/5/8 signalling in human granulosa cells. Mol Cell Endocrinol 2019; 490: 37-46, doi: 10.1016/j.mce.2019.04.003.

20. Chang HM, Y Fang, Liu PP, Cheng JC, X Yang, Leung PC. Connective tissue growth factor mediates growth differentiation factor 8-induced increase of lysyl oxidase activity in human granulosa-lutein cells. Mol Cell Endocrinol 2016; 434: 186-198, doi: 10.1016/j.mce.2016.07.007.

21. Chen W, Chen X, Wang Y, Liu T, Liang Y, Xiao Y, et al. Construction and analysis of IncRNA-mediated ceRNA network in cervical squamous cell carcinoma by weighted gene co-expression network analysis. Med Sci Monit 2019; 25: 2609-2622, doi: 10.12659/MSM.913471.

22. Tu YA, Lin SJ, Chen PL, Chou CH, Huang CC, Ho HN, et al. HSD3B1 gene polymorphism and female pattern hair loss in women with polycystic ovary syndrome. J Formos Med Assoc 2019; 118: 1225-1231, doi: 10.1016/j.jfma.2019. 04.013 .

23. Shao M, Li W. Transcriptional factor regulation network and competitive endogenous RNA (ceRNA) network determining response of esophageal squamous cell carcinomas to neoadjuvant chemoradiotherapy. PeerJ 2019; 7: e6668, doi: $10.7717 /$ peerj.6668.

24. Sun W, Lv J, Duan L, Lin R, Li Y, Li S, et al. Long noncoding RNA $\mathrm{H} 19$ promotes vascular remodeling by sponging let-7a to upregulate the expression of cyclin D1. Biochem Biophys Res Commun 2019; 508: 1038-1042, doi: 10.1016/j.bbrc. 2018.11.185 